\title{
Pathological changes in basement membranes and dermal connective tissue of skin from patients with hereditary cystatin $\mathrm{C}$ amyloid angiopathy
}

\author{
Asbjorg Osk Snorradottir ${ }^{1,2}$, Helgi J Isaksson², Saevar Ingthorsson ${ }^{3,4}$, Elias Olafsson ${ }^{5,6}$, Astridur Palsdottir ${ }^{1}$ and \\ Birkir Thor Bragason ${ }^{1}$
}

Hereditary cystatin $\mathrm{C}$ amyloid angiopathy (HCCAA) is a genetic disease caused by a mutation in the cystatin $\mathrm{C}$ gene. Cystatin $\mathrm{C}$ is abundant in cerebrospinal fluid and the most prominent pathology in HCCAA is cerebral amyloid angiopathy due to mutant cystatin $\mathrm{C}$ amyloid deposition with associated cerebral hemorrhages, typically in young adult carriers. Analyses of post-mortem brain samples shows that pathological changes are limited to arteries and regions adjacent to arteries. The severity of pathological changes at post-mortem has precluded the elucidation of the evolution of histological changes. Mutant cystatin C deposition in carriers is systemic and has, for example, been described in the skin, suggesting similar pathological mechanisms both in the brain and outside of the central nervous system. The aim of this study was to use skin biopsies from asymptomatic and symptomatic carriers to study intermediate events in HCCAA pathogenesis. We found that cystatin C deposition in minimally affected samples was limited to the basement membrane (BM) between the dermis and epidermis. When the deposits were more advanced, they extended to other BM regions in the skin. Our results showed that the immunoreactivity of the BM protein COLIV was increased to a similar extent in all carrier biopsies and cystatin C deposits were in close association with COLIV. The density of fibroblasts in the upper dermis of carrier skin was increased, whereas the distribution of other cell types examined did not differ compared with control biopsies. COLIV and cystatin C immunoreactivity in carrier biopsies was closely associated with the fibroblasts. The results of this study, in conjunction with our previous results regarding pathological BM changes in leptomeningeal arteries of patients, suggest that BM changes are early and important events in HCCAA pathogenesis that could facilitate cystatin C deposition and aggregation.

Laboratory Investigation (2017) 97, 383-394; doi:10.1038/labinvest.2016.133; published online 9 January 2017

Hereditary cystatin C amyloid angiopathy ${ }^{1}$ (HCCAA; Online Mendelian Inheritance in Man entry 105150; also referred to as hereditary cerebral hemorrhage with amyloidosis-Icelandic type (HCHWA-I $)^{2,3}$ ) is a very rare autosomal dominant genetic disease caused by a single base substitution mutation in the gene of the secreted cysteine protease inhibitor cystatin C, CST3. ${ }^{4-6}$ The disease is classified as a cerebral amyloid angiopathy (CAA). The disease-causing mutation has only been found in Iceland (population $~ 332750$ as of 1 January 2016 (Statistics Iceland)) with one exception. ${ }^{7}$ It results in the exchange of leucine for glutamine at amino acid 68 of the protein and will hereafter be referred to as L68Q-CST3. Mutant cystatin C forms amyloid deposits in the walls of cerebral arteries resulting in fatal cerebral hemorrhages in young adults..$^{8-10}$

The arterial pathology underlying cerebral hemorrhages in HCCAA is observed in brain arteries and arterioles both in the white and gray matter and is characterized by extensive changes in the composition and structure of the arterial walls. ${ }^{9}, 10$ In addition to deposition of cystatin $\mathrm{C}$ amyloid, these changes include severe smooth muscle cell loss, accumulation of extracellular matrix proteins, eg, the basement membrane proteins collagen IV (COLIV) and laminin, attenuation of the endothelial layer and associated neuroinflammation in proximity to affected arteries. ${ }^{9,10}$ These changes result in rupture of arteries/arterioles causing cerebral hemorrhages of

${ }^{1}$ Institute for Experimental Pathology at Keldur, University of Iceland, Reykjavik, Iceland; ${ }^{2}$ Department of Pathology, Landspitali University Hospital, Reykjavik, Iceland; ${ }^{3}$ Stem Cell Research Unit, Biomedical Center, University of Iceland, Reykjavik, Iceland; ${ }^{4}$ Department of Laboratory Haematology, Landspitali University Hospital, Reykjavik, Iceland; ${ }^{5}$ Faculty of Medicine, University of Iceland, Reykjavik, Iceland and ${ }^{6}$ Department of Neurology, Landspitali University Hospital, Reykjavik, Iceland

Correspondence: AO Snorradottir, Department of Pathology, Landspitali University Hospital, Baronsstigur, Reykjavik 101, Iceland.

E-mail: aos3@hi.is

Received 27 June 2016; revised 3 November 2016; accepted 6 November 2016 
various degrees and microinfarcts, often presenting as partial paralysis, dementia, and personality changes. The cause of death is usually due to cerebral hemorrhage.

Our previous results ${ }^{10}$ have shown that the extent of the CAA pathology observed in post-mortem brain samples from patients is invariably 'severe' throughout the brain, as per the criteria defined by Vonsattel et al. ${ }^{11}$ The lack of moderate and intermediate pathological changes in such post-mortem samples has made it difficult to decipher the sequence of events leading to the end-stage pathology. Unfortunately, there is no animal model of the disease. Transgenic mice have been produced that express the mutant allele under the control of the neuron-specific Thy-1 promoter ${ }^{12}$ as well as the human CST3 promoter $^{13}$ but neither model developed HCCAA pathology.

HCCAA is a systemic disorder in that cystatin $\mathrm{C}$ is not only deposited within the central nervous system (CNS) of patients but also deposited in peripheral tissues such as lymph nodes, submandibular salivary glands, seminal vesicles, spleen, and skin, suggesting that similar pathological mechanisms are at play in the periphery as in the CNS. ${ }^{8,14-16}$

Our previous results ${ }^{10}$ show that the pathological changes in post-mortem HCCAA brain samples are always spatially associated with arteries, consisting of the damage within the wall itself, the neuroinflammatory reaction to this damage, and in some cases perivascular amyloid and focal deposits in the parenchyma around arteries. We and others ${ }^{10,17}$ have suggested that the cerebral cystatin $\mathrm{C}$ amyloid in HCCAA might originate from cells in the arterial wall itself. However, the extensive vascular damage in cerebral post-mortem samples makes it difficult to conclude about the cell types involved from such tissue samples.

Benedikz et al $^{16}$ described skin deposition of cystatin $\mathrm{C}$ in L68Q-CST3 carriers. The aim of the study described here was to extend on this knowledge and perform a detailed immunohistochemical comparison of skin biopsies from carriers with those from healthy controls to determine whether carrier skin biopsies could provide information about the progression of pathological changes in HCCAA with an emphasis on identifying the cell type responsible for cystatin C tissue deposits.

\section{MATERIALS AND METHODS}

\section{Tissue Samples}

The samples used in this study were acquired by informed consent. The acquisition and use of the samples was according to permits (04-046-S2 and 15-060-S1) from the National Bioethics Committee in Iceland. All samples were processed at the Department of Pathology, Landspitali National University Hospital, Reykjavik, Iceland. Punch skin biopsies (4 mm, central back, one per individual) were obtained from 14 HCCAA L68Q-CST3 carriers (7 males and 7 females, aged $22-67$ years, median $=34$ years; Table 1 ) and 11 healthy controls (4 males and 7 females, aged 28-68 years, median $=42$ years; Table 1 ). Of these 14 carriers, 8 were
Table 1 An overview of the individuals from which skin biopsies were obtained for the study

\begin{tabular}{|c|c|c|c|}
\hline & Age & Gender & Disease status \\
\hline Carrier & 27 & M & $S$ \\
\hline Carrier & 48 & M & $\mathrm{S}$ \\
\hline Carrier & 51 & $F$ & $\mathrm{~S}$ \\
\hline Carrier & 53 & M & $S$ \\
\hline Carrier & 33 & M & $\mathrm{S}$ \\
\hline Carrier & 27 & F & $S$ \\
\hline Carrier & 22 & M & S \\
\hline Carrier & 22 & $F$ & S \\
\hline Carrier & 61 & M & A \\
\hline Carrier & 67 & M & A \\
\hline Carrier & 45 & F & A \\
\hline Carrier & 29 & F & A \\
\hline Carrier & 23 & F & A \\
\hline Carrier & 35 & $\mathrm{~F}$ & A \\
\hline Control & 31 & F & \\
\hline Control & 30 & M & \\
\hline Control & 57 & $F$ & \\
\hline Control & 42 & M & \\
\hline Control & 68 & $\mathrm{~F}$ & \\
\hline Control & 46 & F & \\
\hline Control & 39 & $F$ & \\
\hline Control & 64 & $F$ & \\
\hline Control & 65 & M & \\
\hline Control & 30 & $\mathrm{~F}$ & \\
\hline Control & 28 & M & \\
\hline
\end{tabular}

Abbreviations: $F$, female; $M$, male.

Symptomatic (S) carriers were defined as those who had suffered one or more cerebral hemorrhages leading to hospitalization at the time of biopsy. Asymptomatic (A) carriers had not been hospitalized due to hemorrhages before biopsy. Age refers to the age of the sample donor at the time of sampling.

symptomatic and the remaining 6 asymptomatic (Table 1). The category symptomatic was defined as cerebral hemorrhage leading to documented hospitalization before the time of biopsy. Asymptomatic carriers had not been hospitalized due to cerebral hemorrhage before biopsy. All tissue samples were formalin-fixed and paraffin-embedded. They were cut into $1.5 \mu \mathrm{m}$ sections for hematoxylin and eosin (H\&E) staining and $5 \mu \mathrm{m}$ serial sections for immunohistochemistry, immunofluorescence confocal microscopy, and Congo red staining. H\&E staining was done using standard methods.

\section{Immunohistochemistry}

Sections were de-paraffinized and rehydrated in xylene and ethanol. They were then immunostained using the EnVision 
Table 2 Antibodies used in the study

\begin{tabular}{|c|c|c|c|c|}
\hline Target protein & Company & Species & Pretreatment & Antibody dil. \\
\hline Collagen IV & Sigma, C1926 & Mouse monocl. monocl. & Proteinase $\mathrm{K}^{\mathrm{a}}$ & $1: 500$ \\
\hline Cystatin C & Sigma, HPA013143, (HPA013143) & Rabbit polycl. & None & $1: 100$ \\
\hline E-cadherin & BD, BD610921 & Mouse monocl. & TE buffer, pH 9.0 & $1: 100$ \\
\hline pSMAD2/3 & Santa Cruz, sc-11769 & Rabbit polycl. & TE buffer, pH 9.0 & $1: 200$ \\
\hline p63 & Dako, M7317 & Mouse monocl. & TE buffer, pH 9.0 & $1: 50$ \\
\hline Smooth muscle actin & Abcam, ab7817 & Mouse monocl. & TE buffer, pH 9.0 & $1: 100$ \\
\hline Vimentin & Dako, M7020 & Mouse monocl. & TE buffer, pH 9.0 & $1: 500$ \\
\hline
\end{tabular}

Abbreviations: dil., dilution; monocl., monoclonal; polycl., polyclonal.

The table shows details of the primary antibodies used for immunohistochemistry and immunofluorescence experiments.

aprotease XVII, Sigma, P8038, $1 \mu \mathrm{g} / \mathrm{ml}$.

Detection System Peroxidase/DAB, Rabbit/Mouse kit (Dako, K4065). Pretreatment of samples for epitope retrieval was required for some of the antibodies used (Table 2). Incubations with primary antibodies were performed at room temperature for $30 \mathrm{~min}$. Antibody dilutions (Table 2) were determined in preliminary experiments. After incubation with a primary antibody, sections were incubated with EnVision FLEX/HRP. Sections were washed between steps with Trisbuffered $\mathrm{NaCl}$ solution with Tween 20, pH 7.6 (Dako, S3306). All sections were incubated with 3,3'-diaminobenzidine solution (Dako, K4065) for $10 \mathrm{~min}$. Sections were counterstained with haematoxylin for $5 \mathrm{~min}$ followed by washing with tap water for $10 \mathrm{~min}$. Finally, sections were dehydrated with $100 \%$ ethanol and xylol followed by coverslipping with mounting medium (Pertex, Histolab). Images were acquired with a Nikon Eclipse 50i microscope equipped with a Nikon DS-Fil digital camera and a Nikon Digital Sight DS-U2 camera controller. Image panels were constructed using the GNU Image Manipulation Program (GIMP 2.8.10).

\section{Immunofluorescence Microscopy}

Sections were de-paraffinized and rehydrated in xylene and ethanol. Pretreatment of samples for epitope retrieval was required (Table 2). Sample sections were blocked with 5\% goat serum and stained using two or three primary antibodies in conjunction. Samples were incubated with primary antibodies overnight at $4{ }^{\circ} \mathrm{C}$ except for combinations including the COLIV antibody that were incubated for $1 \mathrm{~h}$ at room temperature. The primary antibody combinations were as follows: (a) anti-cystatin C and anti-COLIV; (b) anti-cystatin $\mathrm{C}$ and anti-vimentin; (c) anti-cystatin $\mathrm{C}$ and anti- $\alpha$ smooth muscle actin; (d) anti-cystatin C and anti-E-cadherin; (e) anti-cystatin $\mathrm{C}$ and anti-p63; (f) anti-cystatin C, anti-COLIV, and anti-vimentin; and (g) anti-vimentin and anti-pSMAD213. The details of the antibodies used are provided in Table 2 . Following incubation with primary antibodies, the sections were further incubated ( $1 \mathrm{~h}$ at room temperature), as required, with DAPI (1:2000, Sigma-Aldrich, D9542) and one or more of the following secondary antibodies: 1:1000 Alexa Fluor 647 goat anti-rabbit IgG (Life Technologies, cat\#A11247), 1:1000 Alexa Fluor 488 goat anti-mouse IgG (Life Technologies, cat\#A11008), 1:1000 Alexa Fluor 488 goat anti-mouse IgG1 (Life Technologies, cat\#A21121), 1:1000 Alexa Fluor 488 goat anti-mouse IgG2a (Life Technologies, cat\#A21131), or 1:1000 Alexa Fluor 546 goat anti-mouse IgG2a (Life Technologies, cat\#A21133). The sections were then washed with buffer and water, air-dried, and coverslipped with Fluoromount ${ }^{\mathrm{TM}}$ (Sigma-Aldrich, F4680) and sealed. Between all the above-mentioned steps in the sample processing, sections were washed with ImmunoFluorescence IMF buffer pH 7.5 (0.1\% TX-100, $0.15 \mathrm{M} \mathrm{NaCl}, 5 \mathrm{mM}$ EDTA, and $20 \mathrm{mM}$ HEPES, $\mathrm{pH}$ 7.5). Immunofluorescence was visualized and captured using an Olympus FV1200 confocal laser scanning microscope. Image panels were constructed using the GNU Image Manipulation Program (GIMP 2.8.10).

\section{Quantification of Cystatin C and COLIV Immunostaining in Skin Biopsies}

Cystatin C and COLIV immunostaining in the patient and control biopsies was quantified by semi-automated image analysis using the ImageJ software (http://rsbweb.nih.gov/,vl. 47) and a method previously described. ${ }^{9}{ }^{910}$ Bright-field images of a section from all individuals were captured on a Nikon Eclipse 50i microscope equipped with a Nikon DS-Fil digital camera and a Nikon Digital Sight DS-U2 camera controller at a resolution of $2560 \times 1920$ pixels using a Nikon $\times 4 / 0.3 \mathrm{NA}$ objective. RGB color images of the sections were imported to ImageJ. On each image, a rectangular $1300 \times 1300$ pixels region of interest (ROI) was defined. The ROI was positioned so that one edge was placed at the periphery of the epidermis ensuring that the ROI extended over the epidermis and well into the dermis. Subsequent processing yielding the \% area coverage of cystatin $\mathrm{C}$ or COLIV immunoreactivity within each ROI was performed as previously described., ${ }^{9,10}$ Statistical analyses on quantitative data were performed with 

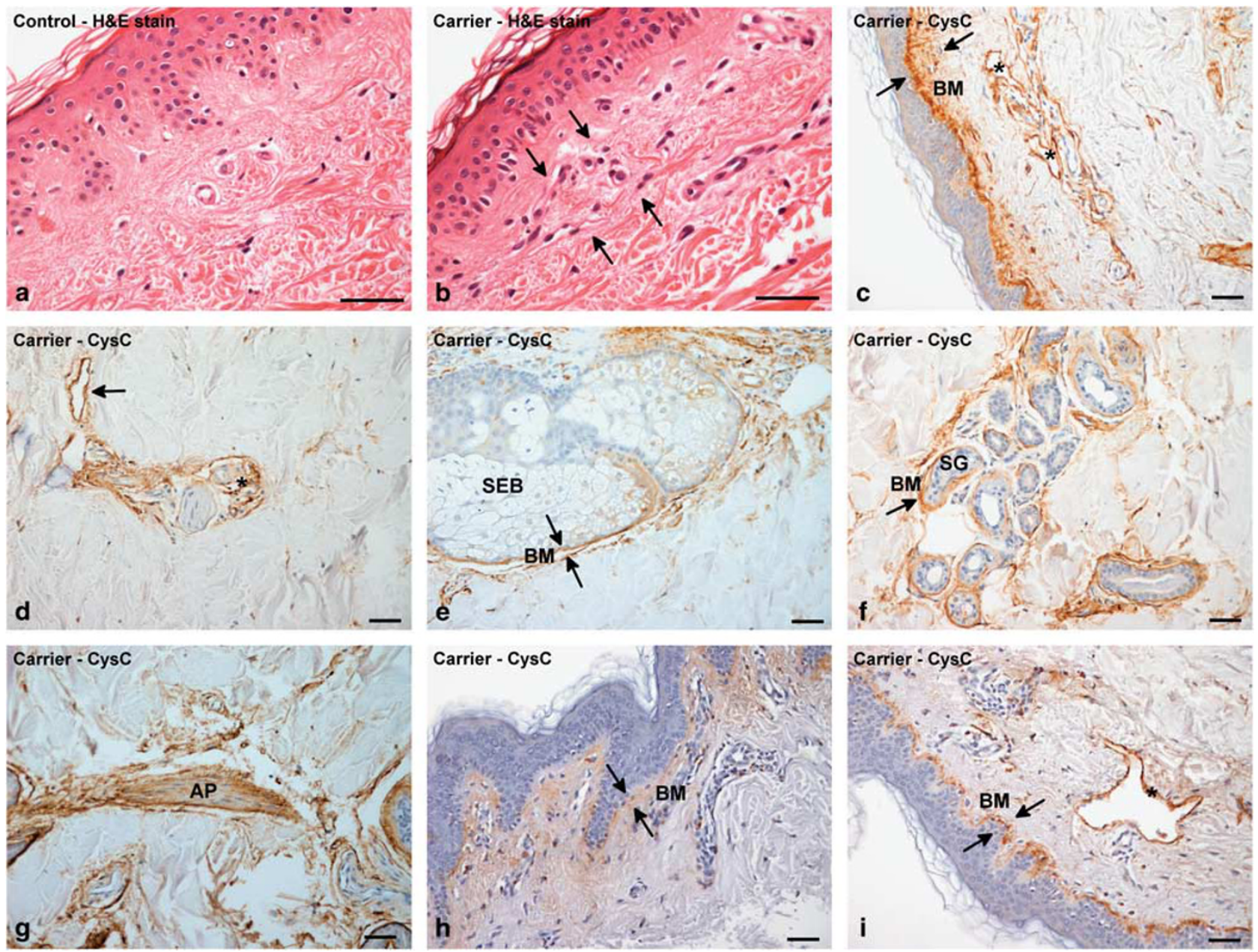

Figure 1 Increased density of cells in the upper dermis of L68Q-CST3 carrier biopsies and the distribution of cystatin C immunoreactivity in biopsies from symptomatic and asymptomatic carriers. (a, b) Hematoxylin and eosin (H\&E) staining of a control biopsy (a) that shows normal epidermis and upper dermis in contrast to a carrier biopsy (b) showing an increased number of cells in the upper dermis (arrows). Examples of cystatin C immunoreactivity in symptomatic carriers: (c) strong cystatin C immunoreactivity in the basement membrane (BM, arrows) between dermis and epidermis (asterisks indicate vessels in the upper dermis with cystatin C immunoreactive BMs), (d) cystatin C immunoreactivity in the BM of a vein (arrow) and arteries (asterisk) in the hypodermis, (e) in the BM (arrows) surrounding a sebaceous gland (SEB) in the dermis, (f) in the BM (arrow) around sweat glands (SG) in the hypodermis, and (g) associated with an arrector pili (AP) muscle. Examples of cystatin C immunoreactvity in asymptomatic carriers: (h) relatively weak and diffuse cystatin C immunoreactivity in the BM between the dermis and epidermis (arrows), and (i) moderate cystatin C deposition in the BM between the dermis and epidermis (arrows) and in the BM of a vessel (asterisk) in the dermis. Scale bars: $50 \mu \mathrm{m}$ in all figures.

GraphPad Instat. The box plot was prepared in StatView v5.0.1.

\section{RESULTS}

\section{Skin Structure in Carriers and Controls}

One skin biopsy per individual (Table 1) was taken from the central back. Examination of H\&E-stained sections did not reveal any major deviations from normal skin tissue structure (epidermis/dermis) in the samples from the carriers compared with controls except that there was an increased cell density in the upper dermis of carrier skin, right below the epidermis (Figures 1a and $\mathrm{b}$ ). H\&E staining revealed mild inflammation in biopsies from two carriers. Focusing on arteries, which are the most affected structures in brain tissue of HCCAA patients, ${ }^{9,10}$ H\&E staining of carrier skin biopsies did not reveal pathological changes in dermal arteries/ arterioles akin to the acellular, homogenous, arterial walls observed in post-mortem brain samples from HCCAA patients.

\section{Cystatin C Deposition in Carrier Biopsies was Associated with Basement Membranes}

Cystatin C immunoreactive deposits were observed in skin biopsies from all the carriers, examples are shown in Figures $1 \mathrm{c}-\mathrm{i}$, whereas biopsies from controls were all negative with the cystatin $\mathrm{C}$ antibody dilution used for the immunohistochemistry (data not shown). Congo red staining of the carrier skin biopsies did not show birefringence under 
polarized light (data not shown), suggesting that the cystatin $\mathrm{C}$ deposits did not contain fully formed amyloid.

The extent of cystatin $\mathrm{C}$ distribution in the skin biopsies differed between symptomatic and asymptomatic carriers in that it was more widespread in the former. In symptomatic carriers, cystatin $\mathrm{C}$ immunoreactivity was observed in the basement membrane between the epidermis and dermis (Figure 1c) extending down into the dermis and up into the epidermis (Figure 1c). It was present in basement membranes of dermal arteries, arterioles and veins (Figures 1c and d), sebaceous glands (Figure 1e), hair follicles, fat/sweat glands (Figure 1f), and arrector pili muscles (Figure 1g).

In contrast to cerebral arteries, in which cystatin C immunoreactivity extends through all layers of the arterial wall and the arteries are often occluded, ${ }^{9,10}$ cystatin C immunoreactivity in dermal vessels was mainly observed in their basement membrane (arteries) but in some cases throughout the entire wall of veins (Figures $1 \mathrm{c}$ and $d$ ), which are minimally affected in the brain; occluded vessels were never observed.

In three of the six asymptomatic carriers, cystatin C immunoreactivity was exclusively observed in the basement membrane between the epidermis and dermis (example shown in Figure $1 \mathrm{~h}$ ) with no immunoreactivity around the skin structures mentioned above with respect to the symptomatic carriers. However, in the remaining three asymptomatic carriers, immunoreactivity was observed around these structures but to a lesser extent than in the symptomatic carriers (Figure 1i).

A quantitative comparison of cystatin $\mathrm{C}$ immunoreactivity between asymptomatic and symptomatic carriers revealed significantly higher levels of immunoreactivity in the latter group $(P<0.0001$, unpaired $t$-test, Figure 2 , left panel). There was no significant correlation between the age of carriers and cystatin C immunoreactivity $(P=0.99$, Pearson $r=-0.003)$.

\section{COLIV Immunoreactivity was Increased in Carrier Biopsies}

The distribution of COLIV immunoreactivity in the biopsies was similar to that of cystatin $\mathrm{C}$ immunoreactivity in the symptomatic carriers, ie, it was present in dermal arteries, arterioles, and veins, in the basement membrane between the epidermis and dermis and around hair follicles, fat/sweat glands, sebaceous glands, and arrector pili muscles (Figures 3a-e).

Quantitative analyses showed that COLIV immunoreactivity was significantly elevated in both asymptomatic and symptomatic carriers compared with controls $(P<0.001$ and $P<0.001$, respectively, ANOVA with Tukey's post test (Figure 2, right panel)). There was, however, no significant difference in COLIV immunoreactivity between the asymptomatic and symptomatic carriers $(P>0.05$, ANOVA with Tukey's post test (Figure 2, right panel)). Within the carriers, there was no significant correlation between the amount of cystatin C and COLIV deposition $(P=0.72$, Pearson

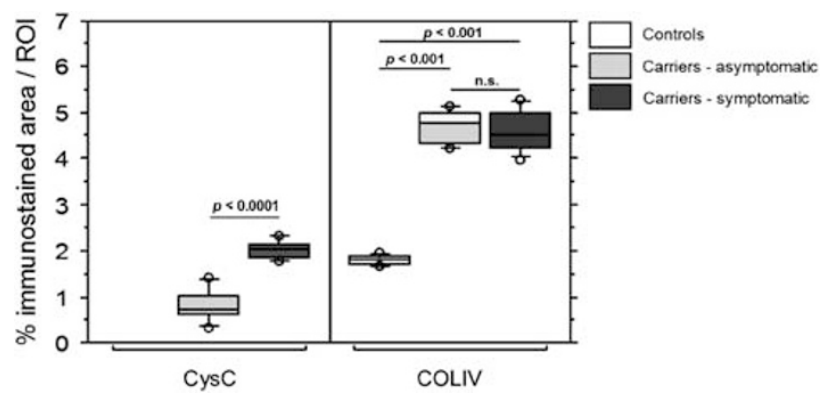

Figure 2 A box and whisker plot that shows the results from quantitative analyses of cystatin C and COLIV in carrier and control biopsies. The whiskers show the 10th percentile and the 90th percentile. Values that fall above the 90th percentile and below the 10th percentile are presented as open circles. Left panel: the plot shows the \% cystatin C immunoreactivity per region of interest (ROI) in skin biopsies of six asymptomatic carriers (light gray box, median (50th percentile) $=0.75$ ) and eight symptomatic carriers (dark gray box, median $=2.04$ ). The $\%$ cystatin $C$ staining per ROI in the carrier biopsies was significantly higher than that in the controls ( $P<0.0001$, unpaired $t$-test). Note: there was no cystatin $C$ immunoreactivity in the control biopsies $(n=11)$ thus no box is shown for the controls. Right panel: the plot shows the \% COLIV immunoreactivity per ROI in biopsies of 11 controls (empty box, median $=1.84$ ), 6 asymptomatic carriers (light gray box, median $=4.77$ ), and 8 symptomatic carriers (dark gray box, median $=4.52$ ). The $\%$ COLIV immunoreactivity per ROI of the asymptomatic and symptomatic carriers was significantly higher than that of the controls $(P<0.001$ and $P<0.001$, respectively (ANOVA with Tukey's post test)). The \% COLIV immunoreactivity per ROI was not significantly (NS) different between asymptomatic and symptomatic carriers ( $P>0.05$ (ANOVA with Tukey's post test)). A full color version of this figure is available at the Laboratory Investigation journal online.

$r=-0.104$ ), suggesting that COLIV immunoreactivity did not increase with cystatin $\mathrm{C}$ deposition, ie, disease progression.

In the biopsies from the carriers, the elevated COLIV immunoreactivity was especially evident in the basement membrane between the epidermis and dermis (Figure 3b). Whereas the distribution of COLIV immunoreactivity in this region of the control biopsies consisted of a thin, relatively well defined, line (Figure 3d), the COLIV immunoreactivity in this area of the carrier biopsies was more extensive and diffuse, ie, it extended to some extent into the epidermis and more extensively down into the dermis, surrounding nuclei in the upper dermis (Figure 3b), indicating changes in basement membrane structure between the epidermis and dermis in the carrier biopsies.

Analysis by confocal immunofluorescence microscopy confirmed the close spatial association between cystatin C and COLIV deposition in the carrier biopsies implied by the standard immunohistochemistry data. This was especially evident in vessel walls and the upper dermis, and was sometimes to the degree of overlap of fluorescent markers (co-localization; Figures $3 \mathrm{e}-\mathrm{g}$ ). Even when the location of the two proteins was not completely co-localized in vessels, cystatin $\mathrm{C}$ was in close proximity slightly peripheral to COLIV (Figure 3g). 

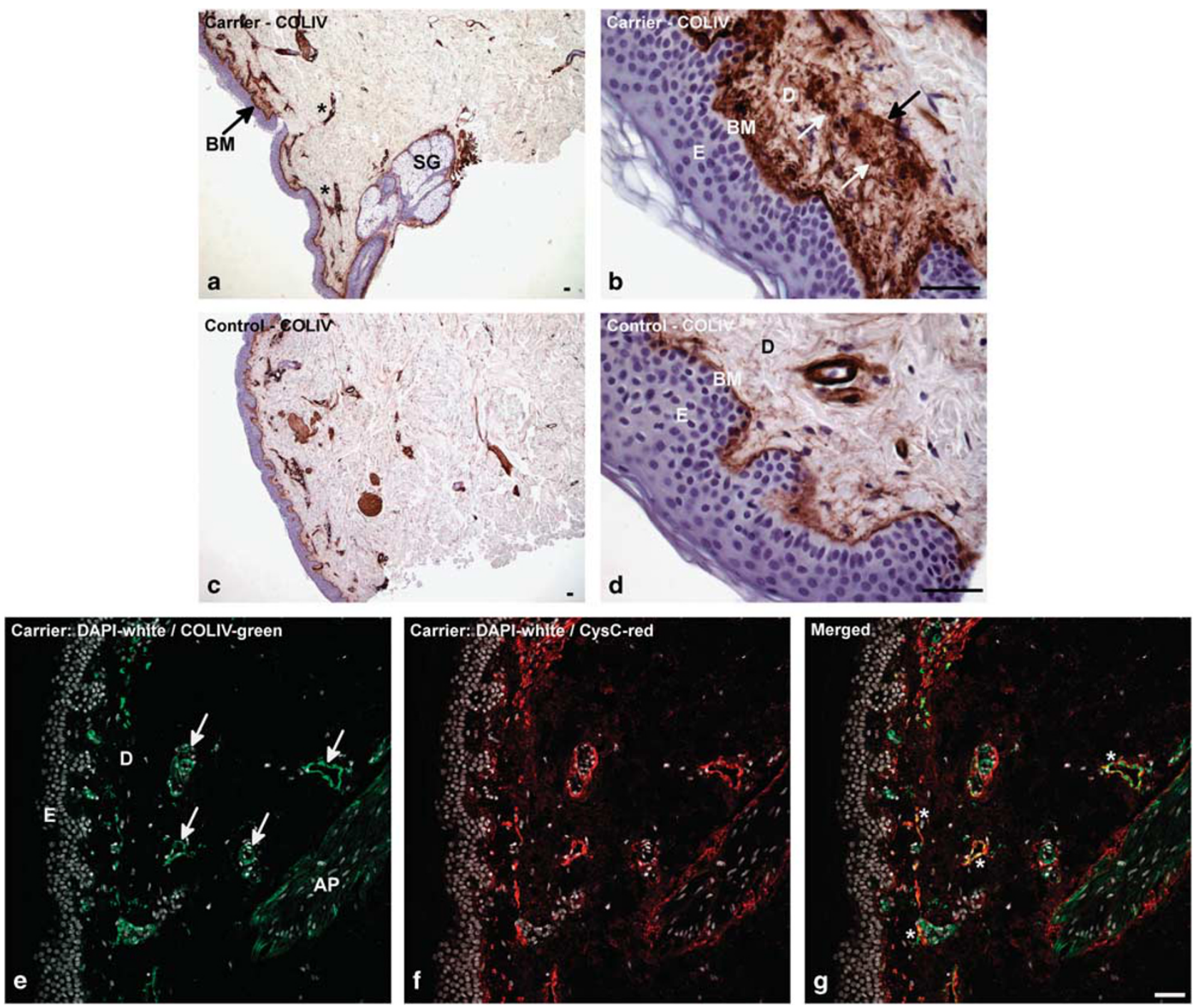

Figure 3 The distribution of COLIV immunoreactivity in skin biopsies from L68Q-CST3 carriers and controls. (a) COLIV immunoreactivity in the skin of a carrier. This low-magnification image shows the overall distribution of COLIV immunoreactivity in the basement membrane (BM, arrow) between the epidermis and dermis as well as in the BM of vessels (examples shown with asterisks) and sebaceous glands (SG). (b) An image taken of the same sample as in a at a higher magnification showing extensive COLIV immunoreactivity in the BM between the dermis (D) and epidermis (E) as well as an increased density of COLIV immunoreactive cells (white arrows) in the upper dermis and thread-like collagen immunoreactive structures (black arrow). (c) A low-magnification image of COLIV immunoreactivity in the skin of a control. The image shows COLIV immunoreactivity in the same areas as in the carrier (a) but to a lesser extent. (d) An image taken of the same sample as in c (same magnification as in b) that shows a thin and well-defined BM between the dermis (D) and epidermis (E) in comparison with that of the carrier in $\mathbf{b}$. This image also shows fewer cell nuclei in the upper dermis of the control, compared with the carrier sample shown in $\mathbf{b}$. (e-g) Immunofluorescence staining of a carrier biopsy: (e) COLIV immunoreactivity in cells of the upper dermis (D), BM of vessels (arrows), and arrector pili (AP) muscle, (f) cystatin C immunoreactivity in the upper dermis and in the BMs of the same vessels and the arrector pili muscle indicated in e. The merged image (g) shows spatial overlap (co-localization) of the green fluorescent label of COLIV and the red fluorescent label of cystatin C giving a yellow color (examples indicated by asterisks). Scale bars: $50 \mu \mathrm{m}$ in all figures.

\section{Cystatin C and COLIV Deposition in Carrier Biopsies was Associated with Fibroblasts}

The distribution of cell types in the biopsies, in relation to the cystatin C and COLIV deposition, was examined using immunohistochemistry and confocal immunofluorescence analyses with antibodies to cell-type markers, ie, antibodies to vimentin, which is a known fibroblast marker, $\alpha$-smooth muscle actin ( $\alpha \mathrm{SMA})$, which is a smooth muscle cell marker, the epithelial cell adhesion molecule E-cadherin, and p63 whose expression is restricted to epithelial cells of stratified epithelia in normal skin.

As mentioned, there was an increase in cell density in the upper dermis of the carrier biopsies. These cells were immunoreactive for vimentin (Figures $4 \mathrm{a}-\mathrm{d}$ ), but not for 

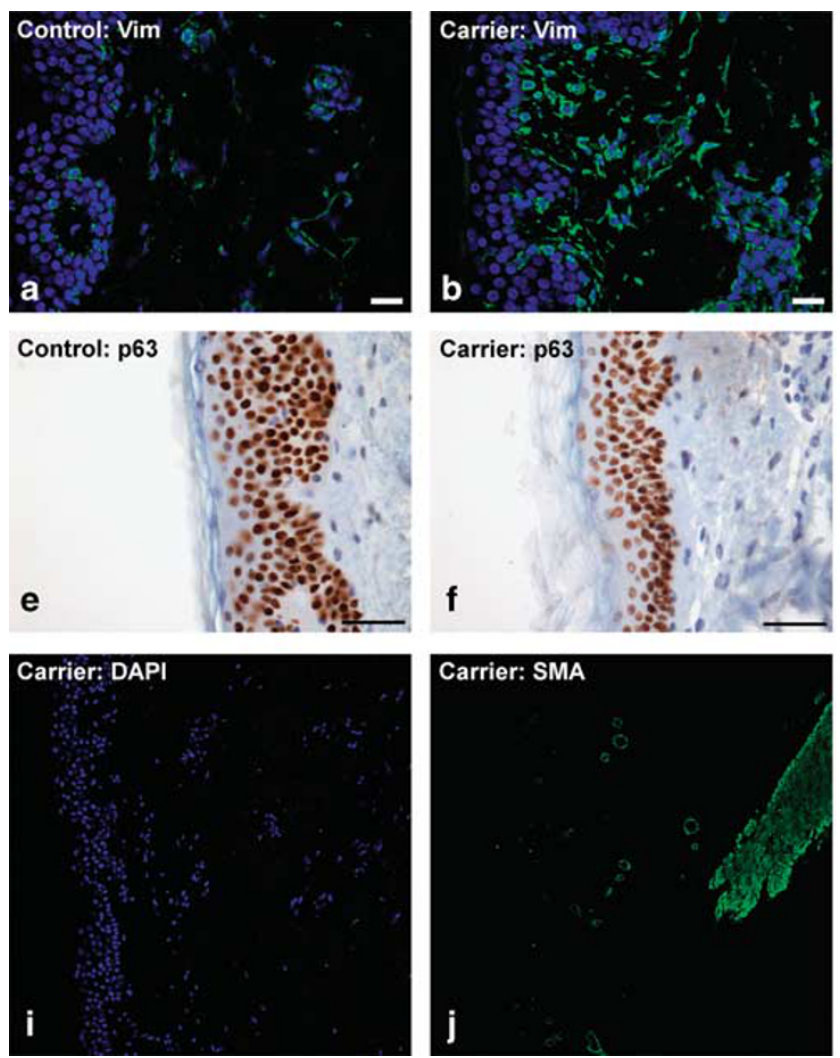
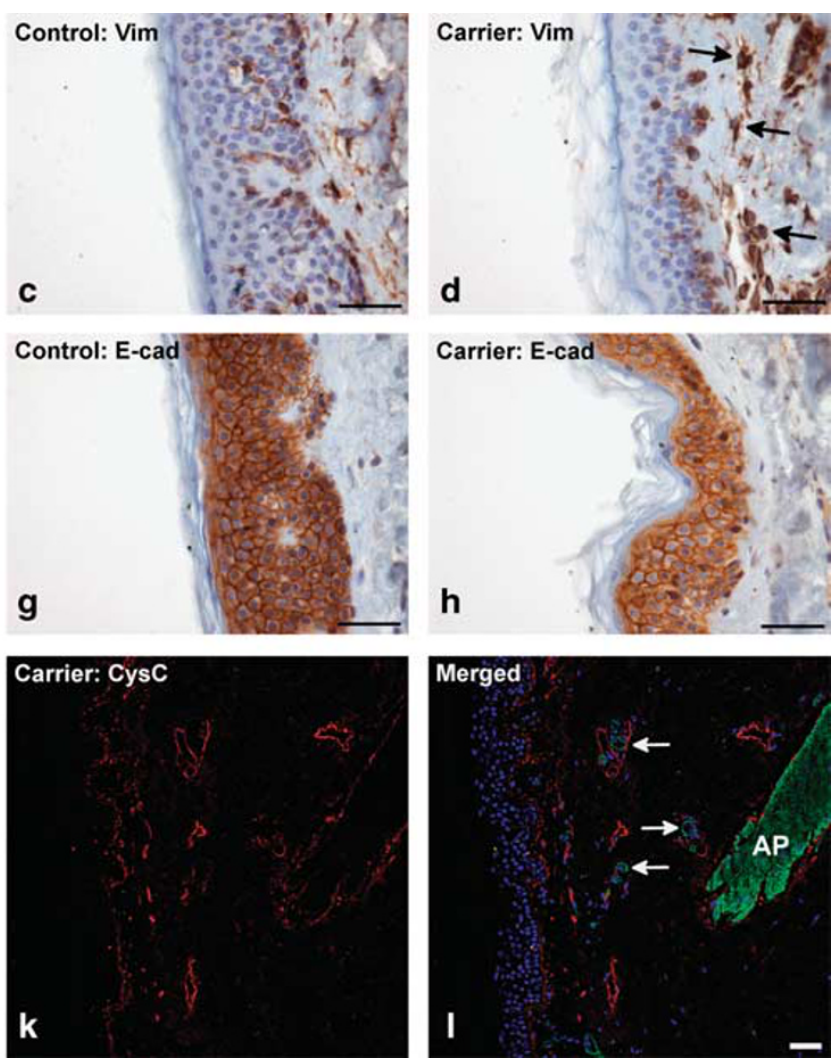

Figure 4 Increased density of vimentin-positive cells in the upper dermis of L68Q-CST3 carriers biopsies. (a) Vimentin (green fluorescence) immunofluorescence staining (blue fluorescence: DAPI) in the upper dermis of a control biopsy. (b) An image of a similar area (as in a) in a carrier biopsy highlighting the increased number of vimentin-positive cells. (c) Vimentin immunoreactivity in the epidermis and upper dermis of a control biopsy. (d) Vimentin immunoreactivity in the epidermis and upper dermis of a carrier showing a different morphology (arrows) of the vimentin-positive cells compared with controls. (e) p63 immunoreactivity in the epidermis and upper dermis of a control biopsy. (f) p63 immunoreactivity in the same area in a carrier biopsy. (g) E-cadherin immunoreactivity in the epidermis and upper dermis of a control biopsy. (h) E-cadherin immunoreactivity in the same area of a carrier biopsy. (i-I) Immunofluorescence staining of a carrier biopsy showing that aSMA immunoreactivity was limited to vessels (I, arrows) and arrector pili (I, AP) muscle in the dermis and did not co-localize with cystatin C. Scale bars: $50 \mu \mathrm{m}$ in all figures.

p63, E-cadherin, or $\alpha$ SMA (Figures $4 \mathrm{e}-1$ ), indicating that they were fibroblasts. E-cadherin and p63 immunoreactivity in carrier and control biopsies was restricted to the epidermis, and $\alpha$ SMA immunoreactivity in the carrier biopsies was limited to the smooth muscle cells of dermal arteries/ arterioles and arrector pili muscle cells (Figure 4l) as in the controls (data not shown). Vimentin-positive cells in the upper dermis were also COLIV positive, as was the case around vessels (Figures $5 \mathrm{a}-\mathrm{c}$ ), accentuating the identity of these cells as fibroblasts.

Morphologically, the fibroblasts in the upper dermis were characterized by an enlarged cell body and the vimentin immunostaining highlighted thicker processes extending from the cell bodies (Figure 4d) compared with vimentinpositive cells in the same area of the controls (Figure 4c). In addition to the upper dermis, cells with a fibroblast marker phenotype were, in general, observed in all cystatin $\mathrm{C}$ and COLIV-positive areas of the carrier biopsies and in some instances cystatin $\mathrm{C}$ immunoreactivity seemed to be intracellular in such cells (Figures $5 \mathrm{a}-\mathrm{c}$ ).

\section{pSMAD2/3 Immunoreactivity in Carrier Biopsies}

The involvement of TGF $\beta$ signaling in the stimulation of extracellular matrix protein production is well documented, ${ }^{18}$ and TGF $\beta$ signaling has been associated with fibrotic connective tissue disorders of the skin. ${ }^{19}$ Relevant to the increased density of fibroblasts and COLIV deposition in the carrier biopsies, TGF $\beta$ has been linked to enhanced proliferation of adult skin fibroblasts. ${ }^{20}$

Phosphorylation of SMAD2/3 is a well documented downstream event in the TGF $\beta$ signaling pathway. ${ }^{18}$ We examined pSMAD2/3 immunoreactivity in the carrier and control biopsies to see whether we could detect evidence of elevated TGF $\beta$ signaling in the carrier biopsies. Our results showed that nuclei of the extended fibroblast population in the carrier biopsies were immunoreactive for pSMAD2/3 (Figures 6a-f). Similar immunoreactivity was, however, also observed in the sparser fibroblast population in the same area of the control skin biopsies (Figures $6 \mathrm{~g}-\mathrm{i}$ ). Thus, although there was a difference in the extent of nuclear pSMAD2/3 immunoreactivity between the carrier and control biopsies, this was 

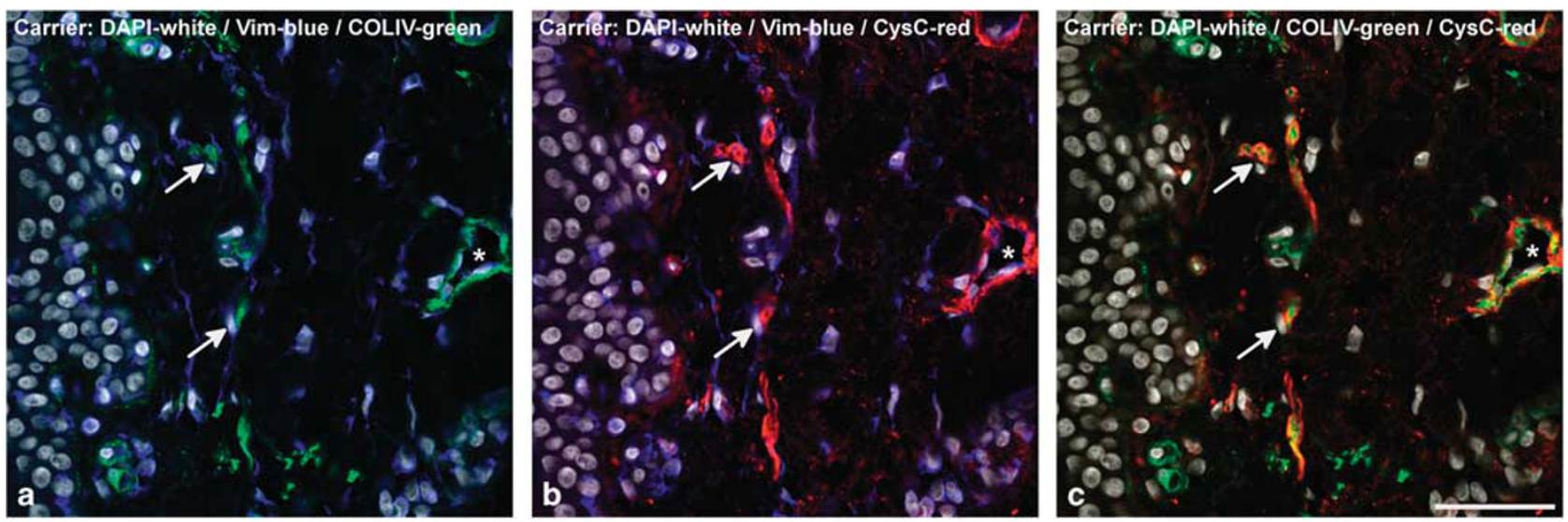

Figure 5 Co-localization of cystatin C, COLIV, and vimentin in skin biopsies of L68Q-CST3 carriers. (a-c) Immunofluorescence staining showing a close association between vimentin, COLIV, and cystatin C immunoreactivity. The arrows and asterisk indicate examples of cells and a vein (respectively) with a spatial overlap of all three fluorescent labels. Scale bars: $50 \mu \mathrm{m}$ in all figures.

mainly due to the denser fibroblast population in the upper dermis of the carrier biopsies compared with the controls.

\section{DISCUSSION}

\section{Cystatin C Deposition in Carrier Biopsies}

Because of its strong cerebral presentation HCCAA is rightly classified as a CAA disorder. However, our results (Palsdottir et $a l^{8}$ and data not shown) and those of others ${ }^{15,16,21}$ show that, although the most advanced pathological changes are found in the CNS, the pathology in HCCAA is not confined to the CNS, but is systemic and characterized by the presence of cystatin $\mathrm{C}$ deposits and Congo red birefringent amyloid in peripheral tissues of patients as well as in the brain. This suggests that the cell types involved in the pathogenesis of HCCAA are not limited to the CNS.

The study described here was performed on a peripheral tissue, skin, which was chosen because cystatin C deposits have been described previously by others in the skin of HCCAA patients and carriers, ${ }^{16}$ due to ease of access and the relatively non-invasive nature of sampling, and also because cell types, similar to those that are present in walls of cerebral arteries and we have suggested ${ }^{10}$ could be responsible for the production of the cystatin $\mathrm{C}$ that forms amyloid, are also present in skin, suggesting that this tissue type could provide data relevant to the understanding of HCCAA pathogenesis.

The location of cystatin C deposits in the skin of L68QCST3 carriers examined in this study concurred with that described previously by Benedikz et al. ${ }^{16}$ Those authors commented that cystatin $\mathrm{C}$ deposition seemed to be more extensive in individuals that had a longer history of the disease. The results of our quantitative analysis reported here confirmed this and showed a statistically significant difference in the quantity of cystatin $\mathrm{C}$ deposition dependent on disease status, ie, symptomatic carriers had significantly higher levels of cystatin $\mathrm{C}$ immunoreactivity than asymptomatic carriers, indicating that the extent of cystatin $\mathrm{C}$ deposition in the skin of carriers to some extent mirrors the progression of the disease in the CNS that can be variable.

Cystatin C deposits in the carrier skin biopsies examined in this study did not show green birefringence after Congo red staining, suggesting that the deposits did not consist of fully formed amyloid that is invariably observed in cerebral arteries of HCCAA patients. This is similar to the results described by Benedikz et al; ${ }^{16}$ however, they observed amyloid-like threads by electron microscopy analyses in some of the skin biopsies they examined from HCCAA patients.

Congo red birefringent cystatin $\mathrm{C}$ amyloid has been described in post-mortem samples from peripheral tissues of patients other than skin, ie, in vessels of lymph nodes, spleen, adrenal cortex, and submandibular salivary glands $s^{8,15,21}$ as well as in the interlobular connective tissue of submandibular salivary glands. These observations show that cystatin $\mathrm{C}$ deposits progress to amyloid form in the periphery as well as in the CNS, and that cystatin $\mathrm{C}$ amyloid in patients is not necessarily confined to vessel walls. Due to the lack of Congo red birefringence in the skin biopsies examined in this study, we concluded that the pathological situation in the carrier biopsies could be described as mild to intermediate and might therefore provide data relevant to early/intermediate events in HCCAA pathogenesis.

There was no correlation between age and cystatin C deposition within the carrier cohort that contained samples from individuals from 22 to 67 years old (Table 1). Our genealogy database shows that the average lifespan of carriers born since 1900 is 31 years (median $=29$ years). ${ }^{22}$ The upper 10th percentile is $\geq 49$ years and the lower 10th percentile $\leq 20$ years. We have arbitrarily defined late-onset carriers as those whose lifespan falls in the top 10th percentile and earlyonset carriers as those that present with symptoms $\leq 35$ years of age. The carrier cohort involved in this study contained samples from both late- and early-onset carriers. Results from our previous work ${ }^{10}$ have led us to conclude that despite the 

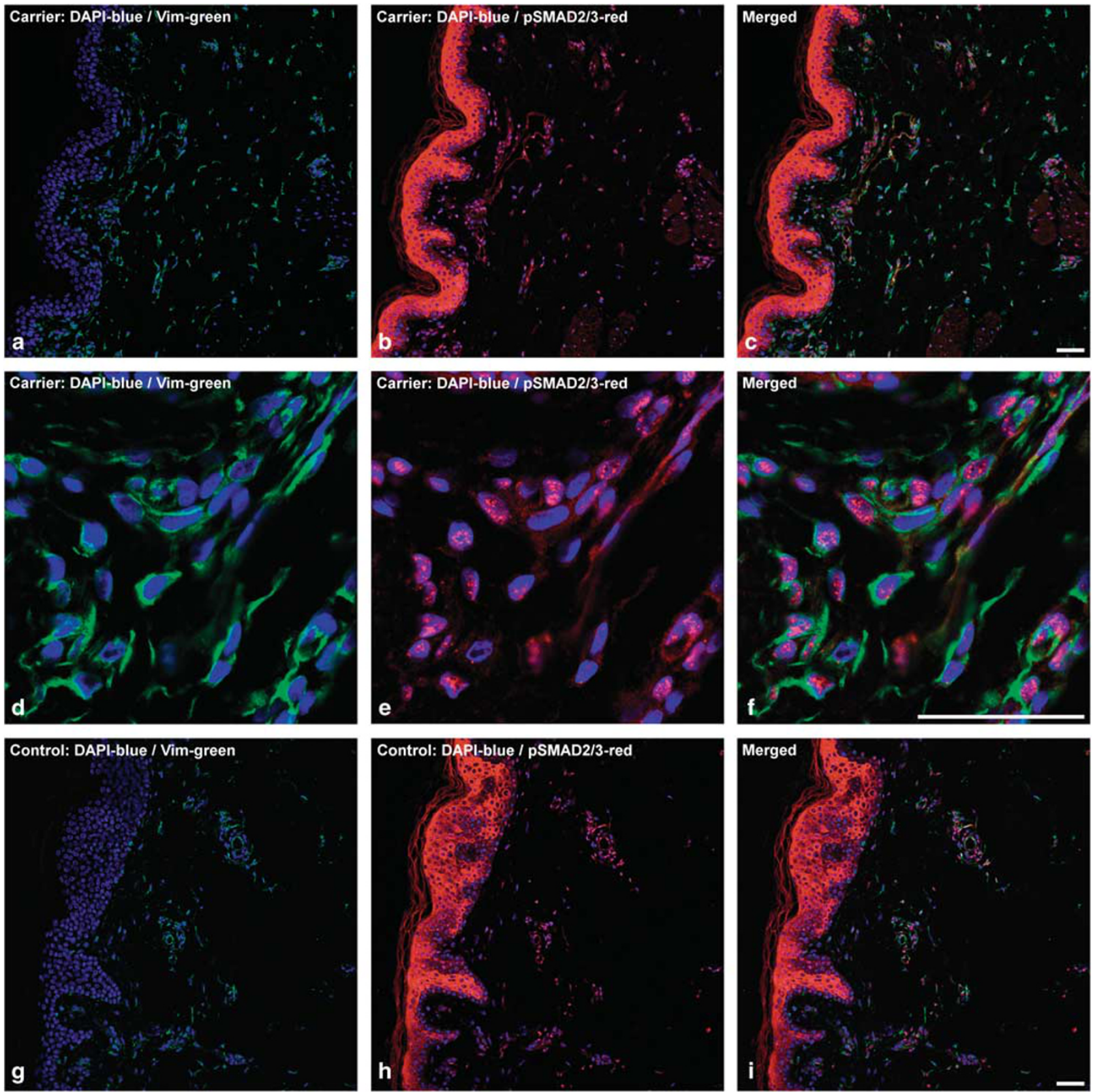

Figure 6 Fibroblasts with nuclear pSMAD2/3 immunoreactivity in skin biopsies of L68Q-CST3 carriers and controls. Immunofluorescence staining (blue: DAPI, green: vimentin, and red: pSMAD2/3) of a carrier (a-f) and control biopsy ( $\mathbf{g}-\mathbf{i})$ showed that vimentin-positive fibroblasts in both carriers and controls displayed pSMAD2/3 immunoreactivity in the nucleus. The difference in pSMAD2/3 immunoreactivity between the carrier and control biopsies was due to the increased density of fibroblasts in the carriers (a) compared with controls (g). (d-f) Images taken of a carrier biopsy at higher magnification showing nuclear pSMAD2/3 immunoreactivity in vimentin-positive fibroblasts. Scale bars: $50 \mu \mathrm{m}$ in all figures.

delayed onset of symptoms, late-onset carriers succumb to the same end-stage pathology as those with an early onset. Therefore, rather than negating the effects of the mutation, there seem to be some factors, internal or external, that can retard the pathological process. Thus, we would not necessarily have expected a correlation between age and cystatin $\mathrm{C}$ deposition in the carrier skin biopsies examined in the present study.

\section{Cystatin C Deposition and the Basement Membrane}

Examination of the skin biopsies from the carriers showed that cystatin $\mathrm{C}$ immunoreactivity in three asymptomatic carriers was exclusively observed in the basement membrane region between the dermis and epidermis, suggesting that initial deposition occurs in that area. In correlation with disease progression, cystatin $\mathrm{C}$ immunoreactivity in the carrier biopsies became more intense and widespread, 
extending down into the upper dermis surrounding structures within the dermis, ie, arteries, veins, glands, and arrector pili muscle, and also extending to some extent up into the epidermis. The deposition, in both symptomatic and asymptomatic carriers, was closely linked to the distribution of the basement membrane collagen COLIV that in turn was closely linked to the expanded population of dermal fibroblasts, whereas there was a lack of a systematic association of cystatin C or COLIV with the markers p63, E-cadherin, or $\alpha$ SMA.

We have previously described extensive basement membrane abnormalities in cerebral arteries/arterioles of HCCAA patients, characterized by deposition of COLIV as well as other extracellular matrix proteins. ${ }^{9}$ The results from that study raised the question whether the deposition was primary, instigating amyloid deposition, or a reactive response to amyloid deposition and/or vascular damage. However, the severity of the pathology in the post-mortem brain samples made it impossible to conclude about the matter.

Our results showed that in contrast to the cystatin C immunoreactivity that differed between asymptomatic and symptomatic carriers, there was what could be termed a 'fixed' elevation of COLIV immunoreactivity in the carrier biopsies tightly associated with cystatin $\mathrm{C}$ deposition and fibroblasts. That is, cystatin $\mathrm{C}$ immunoreactivity increased with disease progression, ie, asymptomatic vs symptomatic, but not COLIV immunoreactivity.

The structure of the basement membrane between the epidermis and dermis is formed by several proteins, such as COLIV, that are contributed by the keratinocytes of the epidermis and dermal fibroblasts. ${ }^{23}$ Some of the proteins are exclusively expressed by either cell type; COLIV is contributed by both. ${ }^{23}$ Our results did not reveal differences in the structure of the epidermis in carrier biopsies compared with controls, but an increase in the density of dermal fibroblasts, which would suggest that the elevation of COLIV immunoreactivity in this region of the carrier biopsies, was associated with the increased density of dermal fibroblasts.

Extracellular matrix proteins, such as COLIV, have been detected in amyloid deposits in Alzheimer's disease (AD) and the CAA disorder Hereditary Cerebral Hemorrhage with Amyloidosis-Dutch type (HCHWA-D), and studies have suggested that they are actively involved in the pathogenesis of these diseases. ${ }^{24-28}$ A recent study by Lepelletier $e t$ a ${ }^{29}$ found that changes in ECM components, ie, COLIV, perlecan and fibronectin, occur in early stages of subclinical AD and that subsequent to the subclinical stage, COLIV expression did not increase further as the disease progressed. Furthermore, they observed correlation between $\mathrm{A} \beta$ and COLIV staining. Our results suggests a similar scenario in HCCAA to that described by Lepelletier $e a^{29}$ for COLIV and $\mathrm{A} \beta$ in $\mathrm{AD}$, ie, COLIV deposition in HCCAA could be an early event in the pathogenesis of the disease, caused by an increased density of dermal fibroblasts, that forms a scaffold facilitating the deposition and aggregation of cystatin $\mathrm{C}$ and subsequent amyloid formation, but does not itself increase as the disease progresses.

Our conclusions based on the results presented here, and our previously reported results, ${ }^{9}$ are that rather than there being a specific cell type responsible for production of the cystatin $\mathrm{C}$ that forms amyloid, a key step in the pathogenesis of HCCAA are basement membrane changes that form a scaffold that promotes deposition and aggregation of cystatin C, which is ubiquitously expressed throughout the body. ${ }^{30}$ In the skin, these basement membrane changes are associated with an increased density of dermal fibroblasts.

\section{Dermal Arteries and Veins in Carrier Biopsies}

Cystatin $\mathrm{C}$ amyloid deposition in brain arteries is invariably accompanied by severe smooth muscle cell loss,, 17 and Wang et $\mathrm{al}^{17}$ suggested that they could be an origin of vascular amyloid in HCCAA. Our results showed that the distribution of $\alpha \mathrm{SMA}$ immunoreactivity did not differ between carrier and control biopsies and they did not indicate a causal relationship between smooth muscle cells and cystatin $\mathrm{C}$ deposition.

It has been reported that solubilized cystatin $\mathrm{C}$ amyloid is toxic to cerebrovascular smooth muscle cells. ${ }^{31}$ Smooth muscle cells in arteries/arterioles are surrounded by basement membrane; therefore, the smooth muscle cell death observed in cerebral arteries and arterioles in HCCAA might be due to the combination of this toxicity and the affinity of cystatin $\mathrm{C}$ for basement membrane.

Interestingly, cystatin $\mathrm{C}$ deposition in the skin biopsies was systematically observed in the walls of veins/venules. Such deposition is rarely observed within the CNS where veins and venules are minimally, or not, affected. ${ }^{10}$ However, immunoreactivity in venous sinuses of the spleen of HCCAA patients has been described. ${ }^{8}$ The reason for this difference between the CNS and the periphery could be the tight association of cystatin C deposition with the basement membrane collagen COLIV and the fact that veins/venules in the periphery differ from those in the CNS in that the latter have very little basement membrane (reviewed by Hawkes et $\left.a b^{32}\right)$.

\section{Similarities with Other Skin Disorders}

There are some similarities between the pathological profile of the L68Q-CST3 biopsies and that described in the literature for connective tissue disorders of the skin, eg, systemic sclerosis, keloids, and hypertrophic scars. Specifically, these disorders are also characterized by excess collagen production and an increased density of dermal fibroblasts (reviewed by Canady et $\mathrm{al}^{19}$ ). However, there are no reports of macroscopic clinical changes in the skin of HCCAA carriers and whereas the other skin disorders are characterized by disturbances in immune parameters and/or inflammation, we did not observe inflammation in the carrier biopsies (except mild inflammation in two cases) and the biopsy sites on the carriers could not be associated with any previous wounds. 
A recent study on keloids described epithelial-tomesenchymal transition (EMT) changes in the disorder. ${ }^{33}$ EMT of the skin is associated with E-cadherin downregulation in skin epidermal cells (epithelia) with concomitant upregulation of, eg, vimentin and p63 in the same cells. ${ }^{34-36}$ As already mentioned, analyses of the distribution of E-cadherin and p63 immunoreactivity in L68Q-CST3 carrier biopsies did not reveal deviations from that observed in the controls. Cells that were immunoreactive for these markers were mainly located in the epidermis and we did not systematically observe an increased induction of mesenchymal characteristics in these cells, ie, vimentin immunoreactivity. Thus, we did not see unequivocal evidence of EMT in the epidermis of the HCCAA carrier samples.

Myofibroblasts are an activated fibroblast type that can be derived by EMT from several cell types, e.g., fibroblasts, and are characterized by upregulated $\alpha \mathrm{SMA}$ expression and enhanced ECM production. ${ }^{37}$ They are generally observed in normal wound healing and in association with classic fibrosis such as fibrotic connective tissue disorders of the skin $^{38,39}$ and various other tissues. ${ }^{37}$ The morphology of the fibroblasts in the upper dermis of carrier biopsies, ie, their enhanced vimentin immunoreactivity as well as the increased density of these cells and their association with COLIV deposition, raised the question whether they were myofibroblasts. Their lack of $\alpha \mathrm{SMA}$ immunoreactivity, however, suggested that they did not fall into this category but does not rule out the possibility that they were protomyofibroblasts. ${ }^{37}$

\section{Cystatin C and TGF $\beta$}

Cystatin $\mathrm{C}$ has been reported to antagonize TGF $\beta$ signaling by binding to the TGF $\beta$ type II receptor (TGF $\beta$ RII) preventing its interaction with $\operatorname{TGF} \beta$ and affecting signaling downstream of the receptor. ${ }^{40,41}$ Therefore, changes in extracellular cystatin C levels could potentially affect signaling through TGF $\beta$ RII. Studies on the processing of mutant and wild-type human cystatin C in primary cells and cell lines have reported intracellular retention, and accumulation, of the mutant protein $^{42-44}$ and extracellular instability due to proteolysis. ${ }^{45}$ Cumulatively, this would be expected to result in lower amounts of extracellular cystatin $\mathrm{C}$ in tissue heterozygous for the L68Q-CST3 mutation compared with wild type, as is indeed the case in cerebrospinal fluid (CSF) of carriers that have less than half the normal value of cystatin $\mathrm{C}$ in their $\mathrm{CSF}^{46}$

TGF- $\beta$ initiates its pro-fibrotic action on fibroblasts by binding to TGF $\beta$ RII whose interaction with TGF $\beta$ RI results in the phosphorylation of the latter and subsequent activation of SMAD2/3 by phosphorylation. Phosphorylated SMAD2/3 then forms a complex with SMAD4 that shuttles to the nucleus and stimulates the transcription of several genes, eg, those of collagen..$^{20,47,48}$

We examined the distribution of pSMAD2/3 in the skin biopsies from the carriers and controls. The results showed that the denser population of fibroblasts in the carrier skin biopsies displayed nuclear immunoreactivity for pSMAD2/3. As expected, due to the normal ongoing nature of cell signaling processes, similar immunoreactivity was also observed in the sparser fibroblast population of the control biopsies.

The results from the pSMAD2/3 immunostaining and the deposition of COLIV and the increased density of fibroblasts in the carrier biopsies observed in this study, coupled with lower extracellular levels of cystatin $\mathrm{C}$ suggested above and the association of cystatin $\mathrm{C}$ with TGF $\beta$ RII, suggest that similar to fibrotic disorders of the skin, ${ }^{19}$ it is possible that TGF $\beta$ could be a factor in the proliferation of dermal fibroblasts in the skin of carriers, and the expansion of COLIV deposition, as a direct consequence of expression of the mutant CST3 allele. This aspect requires further study. However, when compared with other skin diseases with a similar fibrotic phenotype, it would seem that the pathological changes in HCCAA occur at a much slower rate than in, eg, trauma-induced disorders such as keloids.

\section{CONCLUSIONS}

The results of the present study suggest that basement membrane changes facilitate cystatin $\mathrm{C}$ deposition in the skin of L68Q-CST3 carriers. We have previously described advanced basement membrane abnormalities in leptomeningeal arteries of HCCAA patients, ${ }^{9}$ notably, COLIV and laminin deposition, in association with cystatin C amyloid. Taken together, the results reported here and those of the previous study suggest that basement membrane changes are early and important events in HCCAA pathogenesis.

Cystatin $\mathrm{C}$ is ubiquitously expressed throughout the body and it is one of the most abundant proteins in normal CSF. ${ }^{49}$ Basement membrane changes, notably, COLIV deposition, could be pivotal in the pathogenesis of the disease by affecting arterial function in the CNS and, therefore, perivascular drainage of cystatin $\mathrm{C}$ resulting in arterial accumulation of cystatin $\mathrm{C}$ and eventually amyloid formation. This scenario would be similar to that of sporadic CAA, which is associated with reduced perivascular drainage of $\mathrm{A} \beta$ due to age-related arteriosclerosis. $^{50}$

\section{ACKNOWLEDGMENTS}

Our studies on HCCAA have been supported by the Icelandic Centre for Research (RANNIS), the University of Iceland Research Fund, the Memorial fund of Hafdis Kjartansdottir, the Memorial fund of Helga Jonsdottir and Sigurlidi Kristjansson, and the Heilavernd fund. We thank Angelos A. Skodras for technical advice and our collaborators at the Department of Pathology, Landspitali National University Hospital, Iceland, for their assistance with sample processing.

\section{DISCLOSURE/CONFLICT OF INTEREST}

The authors declare no conflict of interest.

1. Jensson O, Gudmundsson G, Arnason A, et al. Hereditary cystatin C (gamma-trace) amyloid angiopathy of the CNS causing cerebral hemorrhage. Acta Neurol Scand 1987;76:102-114.

2. Gudmundsson G, Hallgrimsson J, Jonasson TA, et al. Hereditary cerebral haemorrhage with amyloidosis. Brain 1972;95:387-404. 
3. Cohen $\mathrm{DH}$, Feiner $\mathrm{H}$, Jensson $\mathrm{O}$, et al. Amyloid fibril in hereditary cerebral hemorrhage with amyloidosis (HCHWA) is related to the gastroentero-pancreatic neuroendocrine protein, gamma trace. J Exp Med 1983;158:623-628.

4. Ghiso J, Jensson O, Frangione B. Amyloid fibrils in hereditary cerebral hemorrhage with amyloidosis of Icelandic type is a variant of gammatrace basic protein (cystatin C). Proc Natl Acad Sci USA 1986;83:2974-2978.

5. Levy E, Lopez-Otin C, Ghiso J, et al. Stroke in Icelandic patients with hereditary amyloid angiopathy is related to a mutation in the cystatin C gene, an inhibitor of cysteine proteases. J Exp Med 1989;169: 1771-1778.

6. Palsdottir A, Abrahamson $M$, Thorsteinsson $L$, et al. Mutation in cystatin C gene causes hereditary brain haemorrhage. Lancet 1988;2:603-604.

7. Graffagnino $\mathrm{C}$, Herbstreith $\mathrm{MH}$, Schmechel DE, et al. Cystatin C mutation in an elderly man with sporadic amyloid angiopathy and intracerebral hemorrhage. Stroke 1995;26:2190-2193.

8. Palsdottir A, Snorradottir AO, Thorsteinsson L. Hereditary cystatin C amyloid angiopathy: genetic, clinical, and pathological aspects. Brain Pathol 2006;16:55-59.

9. Snorradottir AO, Isaksson HJ, Kaeser SA, et al. Deposition of collagen IV and aggrecan in leptomeningeal arteries of hereditary brain haemorrhage with amyloidosis. Brain Res 2013;1535:106-114.

10. Osk Snorradottir A, Isaksson HJ, Kaeser SA, et al. Parenchymal cystatin $C$ focal deposits and glial scar formation around brain arteries in hereditary cystatin C amyloid angiopathy. Brain Res 2015;1622: 149-162.

11. Vonsattel JP, Myers RH, Hedley-Whyte ET, et al. Cerebral amyloid angiopathy without and with cerebral hemorrhages: a comparative histological study. Ann Neurol 1991;30:637-649.

12. Kaeser SA, Herzig MC, Coomaraswamy J, et al. Cystatin C modulates cerebral beta-amyloidosis. Nat Genet 2007;39:1437-1439.

13. Mi W, Pawlik M, Sastre M, et al. Cystatin C inhibits amyloid-beta deposition in Alzheimer's disease mouse models. Nat Genet 2007;39: 1440-1442.

14. Lofberg $\mathrm{H}$, Grubb AO, Nilsson EK, et al. Immunohistochemical characterization of the amyloid deposits and quantitation of pertinent cerebrospinal fluid proteins in hereditary cerebral hemorrhage with amyloidosis. Stroke 1987;18:431-440.

15. Thorsteinsson $\mathrm{L}$, Blondal $\mathrm{H}$, Jensson $\mathrm{O}$, et al. Distribution of cystatin $\mathrm{C}$ amyloid deposits in icelandic patients with hereditary cystatin $C$ amyloid angiopathy. In: Isobe T, Shukuro A, Fumiya U, et al. (eds). Amyloid and Amyloidosis. Plenum Publishing Corporation: New York, 1988, pp 585-590.

16. Benedikz E, Blondal H, Gudmundsson G. Skin deposits in hereditary cystatin C amyloidosis. Virchows Arch A Pathol Anat Histopathol 1990;417:325-331.

17. Wang $\mathrm{ZZ}$, Jensson $\mathrm{O}$, Thorsteinsson $\mathrm{L}$, et al. Microvascular degeneration in hereditary cystatin C amyloid angiopathy of the brain. Apmis 1997;105:41-47.

18. Massague J. TGFbeta signalling in context. Nat Rev Mol Cell Biol 2012;13:616-630.

19. Canady J, Karrer S, Fleck M, et al. Fibrosing connective tissue disorders of the skin: molecular similarities and distinctions. J Dermatol Sci 2013;70:151-158.

20. Armatas AA, Pratsinis H, Mavrogonatou E, et al. The differential proliferative response of fetal and adult human skin fibroblasts to TGFbeta is retained when cultured in the presence of fibronectin or collagen. Biochim Biophys Acta 2014;1840:2635-2642.

21. Löfberg $\mathrm{H}$, Grubb AO, Nilsson EK, et al. Immunohistochemical characterization of the amyloid deposits and quantitation of pertinent cerebrospinal fluid proteins in hereditary cerebral hemorrhage with amyloidosis. Stroke 1987;18:431-440.

22. Palsdottir A, Helgason A, Palsson $S$, et al. A drastic reduction in the life span of cystatin C L68Q carriers due to life-style changes during the last two centuries. PLoS Genet 2008;4:e1000099.

23. Marinkovich MP, Keene DR, Rimberg CS, et al. Cellular origin of the dermal-epidermal basement membrane. Dev Dyn 1993;197:255-267.

24. Perlmutter LS. Microvascular pathology and vascular basement membrane components in Alzheimer's disease. Mol Neurobiol 1994;9: 33-40.

25. Kalaria RN, Pax AB. Increased collagen content of cerebral microvessels in Alzheimer's disease. Brain Res 1995;705:349-352.
26. van Duinen SG, Maat-Schieman ML, Bruijn JA, et al. Cortical tissue of patients with hereditary cerebral hemorrhage with amyloidosis (Dutch) contains various extracellular matrix deposits. Lab Invest 1995;73: 183-189.

27. van Horssen J, Otte-Holler I, David G, et al. Heparan sulfate proteoglycan expression in cerebrovascular amyloid beta deposits in Alzheimer's disease and hereditary cerebral hemorrhage with amyloidosis (Dutch) brains. Acta Neuropathol 2001;102:604-614.

28. Zarow C, Barron E, Chui HC, et al. Vascular basement membrane pathology and Alzheimer's disease. Ann NY Acad Sci 1997;826:147-160.

29. Lepelletier FX, Mann DM, Robinson AC et al. Early changes in extracellular matrix in Alzheimer's disease. Neuropathol Appl Neurobiol; e-pub ahead of print 6 November 2016; doi: 10.1111/nan.12295.

30. Abrahamson M, Olafsson I, Palsdottir A, et al. Structure and expression of the human cystatin C gene. Biochem J 1990;268:287-294.

31. Vilhjalmsson DT, Blondal H, Thormodsson FR. Solubilized cystatin C amyloid is cytotoxic to cultured human cerebrovascular smooth muscle cells. Exp Mol Pathol 2007;83:357-360.

32. Hawkes CA, Jayakody N, Johnston DA, et al. Failure of perivascular drainage of beta-amyloid in cerebral amyloid angiopathy. Brain Pathol 2014;24:396-403.

33. Yan L, Cao R, Wang L, et al. Epithelial-mesenchymal transition in keloid tissues and TGF-beta1-induced hair follicle outer root sheath keratinocytes. Wound Repair Regen 2015;23:601-610.

34. Kalluri R, Weinberg RA. The basics of epithelial-mesenchymal transition. J Clin Invest 2009;119:1420-1428.

35. Jonsdottir HR, Arason AJ, Palsson R, et al. Basal cells of the human airways acquire mesenchymal traits in idiopathic pulmonary fibrosis and in culture. Lab Invest 2015;95:1418-1428.

36. Nakamura M, Tokura Y. Epithelial-mesenchymal transition in the skin. J Dermatol Sci 2011;61:7-13.

37. Hinz B. Myofibroblasts. Exp Eye Res 2016;142:56-70.

38. Andrews JP, Marttala J, Macarak E, et al. Keloids: the paradigm of skin fibrosis_Pathomechanisms and treatment. Matrix Biol 2016;51:37-46.

39. Kissin EY, Merkel PA, Lafyatis R. Myofibroblasts and hyalinized collagen as markers of skin disease in systemic sclerosis. Arthritis Rheum 2006;54:3655-3660.

40. Sokol JP, Schiemann WP. Cystatin C antagonizes transforming growth factor beta signaling in normal and cancer cells. Mol Cancer Res 2004;2:183-195.

41. Sokol JP, Neil JR, Schiemann BJ, et al. The use of cystatin C to inhibit epithelial-mesenchymal transition and morphological transformation stimulated by transforming growth factor-beta. Breast Cancer Res 2005;7:R844-R853.

42. Thorsteinsson L, Georgsson G, Asgeirsson B, et al. On the role of monocytes/macrophages in the pathogenesis of central nervous system lesions in hereditary cystatin C amyloid angiopathy. J Neurol Sci 1992;108:121-128.

43. Benedikz E, Merz GS, Schwenk V, et al. Cellular processing of the amyloidogenic cystatin $C$ variant of hereditary cerebral hemorrhage with amyloidosis, Icelandic type. Amyloid 1999;6:172-182.

44. Bjarnadottir M, Wulff BS, Sameni $M$, et al. Intracellular accumulation of the amyloidogenic L68Q variant of human cystatin $\mathrm{C}$ in NIH/3T3 cells. Mol Pathol 1998;51:317-326.

45. Wei L, Berman Y, Castano EM, et al. Instability of the amyloidogenic cystatin $C$ variant of hereditary cerebral hemorrhage with amyloidosis, Icelandic type. J Biol Chem 1998;273:11806-11814.

46. Grubb A, Jensson O, Gudmundsson G, et al. Abnormal metabolism of gamma-trace alkaline microprotein. The basic defect in hereditary cerebral hemorrhage with amyloidosis. N Engl J Med 1984;311: 1547-1549.

47. Macias MJ, Martin-Malpartida P, Massague J. Structural determinants of Smad function in TGF-beta signaling. Trends Biochem Sci 2015;40: 296-308.

48. Fisher GJ, Shao Y, He T, et al. Reduction of fibroblast size/mechanical force down-regulates TGF-beta type II receptor: implications for human skin aging. Aging cell 2016;15:67-76.

49. Irani DN. Cerebrospinal Fluid in Clinical Practice. Elsevier Health Sciences. Saunders Elsevier: Philadelphia, PA (ISBN: 978-1-4160-2908-3), 2009.

50. Weller RO, Boche D, Nicoll JA. Microvasculature changes and cerebral amyloid angiopathy in Alzheimer's disease and their potential impact on therapy. Acta Neuropathol 2009;118:87-102. 